\title{
ABNORMAL CARDIOVASCULAR FINDINGS IN ACUTE PANCREATITIS: ARE THEY ASSOCIATED WITH DISEASE SEVERITY?
}

\author{
Martin A. Chacón-Portillo ${ }^{1}$, Gerardo Payró-Ramírez ${ }^{2}$, Mario C. Peláez-Luna ${ }^{1}$,

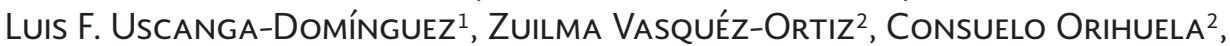 \\ Sophia E. MartíneZ-VÁZqueZ ${ }^{1}$ and Jorge HeRnándeZ-Calleros ${ }^{1}$ \\ ${ }^{1}$ Departments of Gastroenterology and ${ }^{2}$ Cardiolology, Instituto Nacional de Ciencias Médicas y Nutrición Salvador \\ Zubirán, Mexico City, Mexico
}

\begin{abstract}
Background: Acute pancreatitis (AP), a disease that commonly requires in-hospital treatment, has been associated with a high incidence of abnormal cardiovascular findings (ACFs). We conducted a prospective study to explore the association of these findings with severity of the disease. Methods: Adult patients with AP diagnosis were prospectively enrolled in an observational study during an 8-month period in a tertiary care center. AP and its severity were defined according to the Revised Atlanta Classification of AP. Subjects were submitted to electrocardiographic, echocardiographic, and serologic testing during the acute period and a 3-month follow-up. The incidence of ACF was compared between two groups: (1) Mild and (2) moderate/severe cases. Results: Twenty-seven patients (mean age $48 \pm 17$ years) with AP were enrolled; 15 (55\%) had mild and 12 (45\%) had moderate/severe AP. During the acute episode, $67 \%$ had increased pro-brain natriuretic peptide levels; $52 \%$ had abnormal electrocardiographic findings; $48 \%$ had abnormal echocardiographic findings; and $18 \%$ had increased troponin I levels. There was no significant difference in the incidence of ACF between mild and moderate/severe groups. Nineteen patients (70\%) had repeated follow-up testing, and most of the initial ACF did not persist. Conclusion: ACFs occur in an important proportion of patients during AP episodes. Future research should continue to focus in the association of ACFs and the severity of the disease.
\end{abstract}

Key words: Acute pancreatitis. Severity. Electrocardiography. Echocardiography. Organ failure.

\section{INTRODUCTION}

Worldwide, acute pancreatitis (AP) accounts for the highest number of hospital admissions among gastroenterological-related diseases. Improvements in diagnosis and proper referrals have led to an increased proportion of hospitalized patients

\section{Corresponding author:}

*Martin A. Chacón-Portillo

Av. Vasco de Quiroga, 15

Del. Tlalpan

C.P. 14080, Ciudad de México, México

E-mail: martinchaconp@gmail.com having severe AP, which is still associated with a high mortality ${ }^{1}$.

Abnormal cardiovascular findings (ACF) during AP episodes have been described since the early $20^{\text {th }}$ century ${ }^{2}$. Approximately $50 \%$ of AP cases present electrocardiogram (EKG) changes with the most common
Received for publication: 06-08-2017

Approved for publication: 17-09-2017

doi: $10.24875 /$ RIC.17002361 
being T-wave flattening and ST-segment depression ${ }^{3}$. Hemodynamic changes and pericardial changes have also been consistently described ${ }^{4}$.

Increased troponin and brain natriuretic peptide (BNP) serum levels have recently been associated with increased AP severity ${ }^{5,6}$. Of note, BNP $5,7,8$ and troponin 9 serum concentrations are highly specific for cardiac damage.

Although frequently described, the pathophysiology of ACFs during AP episodes is poorly understood. Mechanisms such as myocardial ultrastructural disturbances $^{10}$, release of pancreatic proteolytic enzymes ${ }^{11}$, hypovolemia, cytokines ${ }^{4}$, electrolyte disturbances ${ }^{3,4}$, and hemodynamic instability have been previously proposed $^{12}$.

A recent prospective cohort study reported an association between increased mortality in AP and the presence of QTc-interval prolongation, pericardial effusion, and diastolic dysfunction ${ }^{13}$. There are no reports in the literature, as far as we are aware, of studies assessing the relationship of ACFs with the severity of AP.

The objective of the present study was to analyze the association between ACFs and AP severity.

\section{METHODS}

The present study was conducted between March 2015 and October 2015 at a tertiary care center. All consecutive patients with an AP diagnosis were prospectively enrolled after an informed consent was obtained. The Institutional Review Board approved the study. The institutional standard of care was followed in all patients.

The study included patients with age group of 18 years old or older admitted during the first 14 days of AP course. Patients with a past medical history of cardiovascular disease, chronic renal failure, or pregnancy were excluded from the study.

Demographic and clinical data were collected prospectively including EKG, echocardiogram (ECO), and venous blood sample performance to measure troponin I (Tnl) and pro-BNP levels at the acute and follow-up visit ( $\sim 3$ months after discharge).
Venous blood sampling, EKG, and ECO were performed at a median of 2 days $(0-10), 2$ days $(0-7)$, and 3 days (0-12), respectively, from disease onset. All findings outside of standardized parameters were noted. A follow-up visit occurred at a median of 3.4 months $(2.4-6.1)$ after AP onset in $70 \%(n=19)$ of patients.

A blinded senior cardiologist (G.P.R) reviewed all EKG studies. Of note, isolated sinus tachycardia is described but not included in the comparative analyses between AP severities. Two senior echocardiographists (Z.V.O and $\mathrm{C} . \mathrm{O}$ ) reviewed all ECO results.

AP severity and diagnosis were defined per the Revised Atlanta Classification of $\mathrm{AP}^{14}$, which has been extensively validated ${ }^{15}$. Considering the fact that both moderately severe and severe APs have a worse prognosis than mild $\mathrm{AP}^{16,17}$, we classified AP as mild or moderate/severe to increase the sensitivity of the study.

\section{Statistical analysis}

Data analyses were performed using $\chi^{2}$, Fisher exact test, Student's $t$-test, or Mann-Whitney test, accordingly. A two-sided $p<0.05$ was considered statistically significant. All analyses were performed using the SPSS version 22 (SPSS for Mac; IBM, Chicago, Illinois, USA). Continuous variables are presented as median (range) or mean ( \pm standard deviation), as appropriate, and qualitative variables as frequencies (percentages), unless otherwise specified.

\section{RESULTS}

A total of 27 patients with AP were included in the study. Their mean age was 48 years \pm 17 and 17 (63\%) were female. They were admitted at a median of 1 day $(0-7)$ of disease course. The demographic characteristics are shown in table 1 . Of note, vasopressors were not used; the therapeutic interventions were intravenous fluid replacement ( $n=27,100 \%$ ), oxygen therapy $(n=6,22 \%)$, antibiotics $(n=7,26 \%)$, and retrograde cholangiopancreatography $(n=6,22 \%)$. The median length of hospital stay was 6 days (2-32). There were no in-hospital deaths; one patient died after discharge, unrelated to the pancreatic event. 
Fourteen (52\%) patients had $\geq 1$ abnormal EKG finding after excluding isolated tachycardia $(n=6,22 \%)$. The incidence of EKG findings and their association with AP severity is shown in table 2 . Thirteen ( $48 \%$ ) patients had $\geq 1$ abnormal ECO finding. The ECO findings and their association with AP severity are shown in table 3 . A total of $5(26 \%)$ and $6(32 \%)$ cases had EKG and ECO abnormalities at follow-up, respectively.

Serologic abnormalities in our cohort were present in more than $50 \%$ of the cases. The relationship between serological markers and AP severity is shown in Table 4. Of note, in 1 patient (5\%), pro-BNP levels were elevated $(152 \mathrm{pg} / \mathrm{mL})$ at follow-up.
In total, 21 subjects (77\%) had evidence of electrolyte disturbances; the most prevalent were hypophosphatemia ( $n=15,55 \%)$, hypomagnesemia $(n=13,48 \%)$, hyponatremia $(n=7,25.9 \%)$, hypokalemia $(n=7,25.9 \%)$, and hypocalcemia $(n=6,22 \%)$. Hyponatremia was associated with QTc-interval prolongation ( $p=0.013$ ), and hypokalemia was associated with sinus tachycardia $(p=0.028)$.

\section{DISCUSSION}

The present prospective exploratory study is, to the best of our knowledge, the first to evaluate the

Table 1. Demographic and clinical characteristics of patients with AP

\begin{tabular}{|c|c|c|c|c|}
\hline Characteristics & $\begin{array}{l}\text { Mild AP } \\
(n=15)\end{array}$ & $\begin{array}{c}\text { Moderate/severe AP } \\
(n=12)\end{array}$ & $\begin{array}{c}\text { Total } \\
(n=27)\end{array}$ & $\mathrm{p}$ value \\
\hline Female, n (\%) & $9(60)$ & $8(66.7)$ & $17(63)$ & 0.722 \\
\hline Age, median (range) & $43(21-69)$ & $56(22-79)$ & $51(21-79)$ & 0.373 \\
\hline \multicolumn{5}{|l|}{ Etiology, n (\%) } \\
\hline Gallstones & $9(60)$ & $10(83.3)$ & $19(70)$ & 0.56 \\
\hline Dyslipidemia & $2(13)$ & $1(8.3)$ & $3(11)$ & \\
\hline Post-ERCP & $3(20)$ & $1(8.3)$ & $4(15)$ & \\
\hline Idiopathic & $1(7)$ & $0(0)$ & $1(4)$ & \\
\hline \multicolumn{5}{|l|}{ Comorbidity, n (\%) } \\
\hline Tobacco & $5(33)$ & $1(8)$ & $6(22)$ & 0.182 \\
\hline Dyslipidemia & $5(33)$ & $4(33)$ & $9(33)$ & 0.999 \\
\hline Hypertension & $4(27)$ & $3(25)$ & $7(26)$ & 0.999 \\
\hline Alcohol & $3(20)$ & $1(8)$ & $4(15)$ & 0.605 \\
\hline Cocaine & $1(7)$ & $0(0)$ & $1(4)$ & 0.999 \\
\hline Diabetes mellitus & $2(13)$ & $4(33)$ & $6(22)$ & 0.357 \\
\hline Hypothyroidism & $2(13)$ & $1(8)$ & $3(11)$ & 0.999 \\
\hline Cholangitis & $3(20)$ & $5(42)$ & $8(30)$ & 0.398 \\
\hline Choledocolithiasis & $1(7)$ & $2(17)$ & $3(11)$ & 0.569 \\
\hline Pneumonia & $1(7)$ & $2(17)$ & $3(11)$ & 0.569 \\
\hline
\end{tabular}

AP: acute pancreatitis; ERCP: endoscopic retrograde cholangiopancreatography

Table 2. Abnormal electrocardiographic findings in patients with acute pancreatitis

\begin{tabular}{|c|c|c|c|c|c|}
\hline Electrocardiographic findings & $\begin{array}{c}\text { Mild AP } \\
\mathrm{n}=15(\%)\end{array}$ & $\begin{array}{c}\text { Moderate/severe AP } \\
n=12(\%)\end{array}$ & $\begin{array}{c}\text { Total } \\
\mathrm{n}=\mathbf{2 7}(\%)\end{array}$ & $\mathrm{p}$ value & $\begin{array}{l}\text { Follow-up } \\
n=19(\%)\end{array}$ \\
\hline Sinus tachycardia & $3(20)$ & $3(25)$ & $6(22)$ & - & $1(5)$ \\
\hline Bradycardia & $1(7)$ & $3(25)$ & $4(15)$ & 0.294 & $0(0)$ \\
\hline QRS prolongation & $0(0)$ & $1(8)$ & $1(4)$ & 0.444 & $0(0)$ \\
\hline QTc prolongation & $0(0)$ & $3(25)$ & $3(11)$ & 0.075 & $0(0)$ \\
\hline RBBB & $0(0)$ & $1(8)$ & $1(4)$ & 0.444 & $2(11)$ \\
\hline LAFB & $1(7)$ & $2(17)$ & $3(11)$ & 0.569 & $2(11)$ \\
\hline Non-specific repolarization changes & $1(7)$ & $2(17)$ & $3(11)$ & 0.569 & $2(11)$ \\
\hline T-wave inversion & $0(0)$ & $1(8)$ & $1(4)$ & 0.99 & $0(0)$ \\
\hline ST depression & $1(7)$ & $0(0)$ & $1(4)$ & 0.99 & $0(0)$ \\
\hline ST elevation & $0(0)$ & $1(8)$ & $1(4)$ & 0.444 & $0(0)$ \\
\hline LA abnormality & $0(0)$ & $1(8)$ & $1(4)$ & 0.444 & $0(0)$ \\
\hline Atrial fibrillation & $0(0)$ & $1(8)$ & $1(4)$ & 0.444 & $0(0)$ \\
\hline Inferior QS complex & $0(0)$ & $1(8)$ & $1(4)$ & 0.444 & $1(5)$ \\
\hline
\end{tabular}

AP: acute pancreatitis; LA: left atrium; RBBB: right bundle branch block; LAFB: left anterior fascicular branch block 
Table 3. Abnormal echocardiographic findings in patients with acute pancreatitis

\begin{tabular}{|c|c|c|c|c|c|}
\hline $\begin{array}{l}\text { Echocardiographic } \\
\text { findings }\end{array}$ & $\begin{array}{c}\text { Mild AP } \\
\mathrm{n}=15(\%)\end{array}$ & $\begin{array}{c}\text { Moderate/severe AP } \\
n=12(\%)\end{array}$ & $\begin{array}{c}\text { Total } \\
\mathrm{n}=27(\%)\end{array}$ & $p$ value & $\begin{array}{l}\text { Follow-up } \\
\mathrm{n}=19(\%)\end{array}$ \\
\hline Right ventricle dilation & $0(0)$ & $1(8)$ & $1(4)$ & 0.440 & $0(0)$ \\
\hline Diastolic dysfunction & $2(13)$ & $1(8)$ & $3(11)$ & 0.99 & $1(5)$ \\
\hline Pulmonary hypertension & $4(27)$ & $5(42)$ & $9(33)$ & 0.448 & $2(11)$ \\
\hline Tricuspid regurgitation & $3(20)$ & $2(17)$ & $5(19)$ & 0.999 & $1(5)$ \\
\hline Mitral regurgitation & $2(13)$ & $0(0)$ & $2(7)$ & 0.487 & $0(0)$ \\
\hline Aortic insufficiency & $2(13)$ & $1(8)$ & $3(11)$ & 0.999 & $3(16)$ \\
\hline Left atrial dilation & $0(0)$ & $1(8)$ & $1(4)$ & 0.444 & $0(0)$ \\
\hline Hypokinesis & $1(7)$ & $0(0)$ & $1(4)$ & 0.999 & $0(0)$ \\
\hline Pulmonary insufficiency & $0(0)$ & $0(0)$ & $0(0)$ & - & $2(11)$ \\
\hline Systemic ejection fraction & $65 \pm 6 \%$ & $68 \pm 7 \%$ & $66 \pm 6 \%$ & 0.342 & $67 \pm 6 \%$ \\
\hline
\end{tabular}

AP: acute pancreatitis.

Table 4. Increased serological markers of cardiac damage in patients with acute pancreatitis

\begin{tabular}{lccr}
\hline Serological markers & Mild AP $(\mathrm{n}=15)$ & Moderate/severe AP $(\mathrm{n}=12)$ & Total $(\mathrm{n}=27)$ \\
\hline $\begin{array}{l}\text { Tnl }(\mathrm{ng} / \mathrm{mL}) \\
\text { Increased }\end{array}$ & $2(13)$ & $3(25)$ & $5(19)$ \\
$\begin{array}{l}\text { Pro-BNP }(\mathrm{pg} / \mathrm{mL}) \\
\quad \text { Increased }\end{array}$ & $8(53)$ & $6(50)$ & 0.628 \\
$\quad$ Levels (standard desviation) & $103.2( \pm 73.4)$ & $126.3( \pm 75.3)$ & $14(52)$ \\
\hline
\end{tabular}

AP: acute pancreatitis; Pro-BNP: Pro-brain natriuretic peptide; Tnl: troponin I.

relationship between acute serologic, EKG, and ECO ACFs and the severity of AP based on the Revised Atlanta Classification of $\mathrm{AP}^{14}$. Current clinical prognostic scoring systems have a poor positive predictive value for persistent organ failure in AP. The ability of clinicians to predict severity cannot improve until new approaches to disease severity classification are attempted ${ }^{18}$. Early identification of patients at risk of developing organ failure should be a priority for clinicians to intervene accordingly ${ }^{18,19}$.

The present study has numerous significant findings. First, our findings support the premise that there is a high incidence of ACFs in AP patients ${ }^{3,4}$. Contrary to the findings of Rubio-Tapia et al., bradycardia and sinus tachycardia were the most prevalent EKG findings ${ }^{3}$. However, our results support their conclusion which associates electrolyte disturbances with EKG abnormalities during AP episodes. This further demonstrates the need for a prospective study with a larger sample size to ascertain the incidence distribution of EKG findings in AP patients.

To the best of our knowledge, this is the first study that reports the breadth of ECO findings (Table 3 ) that occur during AP episodes and at follow-up. Nadkarni et al. ${ }^{13}$ reported abnormal ECO findings solely in the acute episode.

More than $50 \%$ of our cohort presented with increased pro-BNP serum levels. However, increased levels were not associated with a higher AP severity, unlike a previous study in a larger sample size but using different AP severity criteria ${ }^{5}$. Only 1 patient $(5.2 \%)$ in our cohort had increased Pro-BNP levels at follow-up. This finding supports the premise that the acute inflammatory state that the body undergoes might cause the release of pro-BNP20.

We report a high prevalence of $\mathrm{Tnl}$ increase without an association to disease severity. A previous group had found an association with increased AP severity, although they analyzed troponin subtype $T$ instead of I and used an older version of the Atlanta criteria ${ }^{6}$. It is important for clinicians to take into account the high prevalence of $\mathrm{Tnl}$ elevation in AP cases, $18.5 \%$ in our cohort, which along with the EKG changes could be difficult to distinguish from an acute coronary syndrome ${ }^{20}$.

Although previously reported by Nadkarni et al. ${ }^{13}$, our study did not find a relationship between AP mortality and QTc-interval prolongation, pericardial effusion, and 
diastolic dysfunction. Of note, their group had a relatively high mortality (15.3\%) compared to our cohort, which did not have in-hospital deaths. In terms of AP severity, QTc-interval prolongation was not associated $(p=0.075)$. However, all the 3 patients who presented QTc-interval prolongation were classified as moderate/severe cases. Taking into account the results of the formerly mentioned group ${ }^{13}$ and the known risk of mortality with higher AP severity ${ }^{16,17}$, the lack of significance may be secondary to our sample size. Two of these patients had a follow-up EKG and did not have QTc-interval prolongation, ruling out its presence due to an unknown underlying primary cardiac condition.

Our study is not without limitations. We investigated a relatively small sample from a single center and had a 30\% loss to follow-up, which could be causing a significant bias in our results ${ }^{21,22}$. Loss to follow-up was mainly attributed to a failure to communicate with patients to schedule follow-up testing. Two additional patients refused or were unable to continue follow-up, and the late death was also considered lost to follow-up. Not all testing was performed simultaneously. For this reason, the ACFs described may represent different stages of a disease known for its dynamism ${ }^{16,17}$. However, as shown by the median time to each type of study, most studies ( $52 \%$ ) were performed within the first 3 days of disease progression.

In summary, ACFs occur in an important proportion of patients during AP episodes. Due to the study's limitations, the number of conclusions that can be drawn from our results is restricted. Nonetheless, this exploratory study generates a hypothesis that merits confirmation by the future studies with a larger sample size to obtain an appropriate statistical power of the findings.

\section{REFERENCES}

1. Agarwal S, George J, Padhan RK, et al. Reduction in mortality in severe acute pancreatitis: A time trend analysis over 16 years. Pancreatology. 2016;16:194-9.

2. Drummond J. Cardiac abnormalities of abdominal origin. S Afr Med J. 1934;8:520-6.

3. Rubio-Tapia A, García-Leiva J, Asensio-Lafuente E, Robles-Díaz G, Vargas-Vorácková F. Electrocardiographic abnormalities in patients with acute pancreatitis. J Clin Gastroenterol. 2005; 39:815-8.

4. Yegneswaran B, Kostis JB, Pitchumoni CS. Cardiovascular manifestations of acute pancreatitis. J Crit Care. 2011;26:225. e11-8.

5. Bugdaci MS, Oztekin E, Kara E, Koker I, Tufan A. Prognostic value of increased $B$ type natriuretic peptide in cases with acute pancreatitis. Eur J Intern Med. 2012;23:e97-e100.

6. Pezzilli R, Barassi A, lammarino MT, d'Eril GM. Is troponin T a useful marker of myocardial damage in acute pancreatitis? A prospective time course study. Dig Liver Dis. 2013;45:347-8.

7. Mair J, Friedl W, Thomas S, Puschendorf B. Natriuretic peptides in assessment of left-ventricular dysfunction. Scand J Clin Lab Invest Suppl. 1999;230:132-42.

8. Nishikimi T, Minamino N, Ikeda M, et al. Diversity of molecular forms of plasma brain natriuretic peptide in heart failure-different proBNP-108 to BNP-32 ratios in atrial and ventricular overload. Heart. 2010;96:432-9.

9. Thygesen K, Mair J, Katus $\mathrm{H}$, et al. Recommendations for the use of cardiac troponin measurement in acute cardiac care. Eur Heart J. 2010;31:2197-204.

10. Saulea A, Costin S, Rotari V. Heart ultrastructure in experimental acute pancreatitis. Rom J Physiol. 1997;34:35-44.

11. Albrecht CA, Laws FA. ST segment elevation pattern of acute myocardial infarction induced by acute pancreatitis. Cardiol Rev. 2003;11:147-51.

12. Khairy P, Marsolais P. Pancreatitis with electrocardiographic changes mimicking acute myocardial infarction. Can J Gastroenterol. 2001:15:522-6.

13. Nadkarni N, Bhasin DK, Rana SS, et al. Diastolic dysfunction, prolonged QTc interval and pericardial effusion as predictors of mortality in acute pancreatitis. J Gastroenterol Hepatol. 2012;27:1576-80.

14. Banks PA, Bollen TL, Dervenis C, et al. Classification of acute pancreatitis--2012: Revision of the Atlanta classification and definitions by international consensus. Gut. 2013;62: 102-11.

15. Talukdar R, Bhattacharrya A, Rao B, Sharma M, Reddy DN. Clinical utility of the revised Atlanta classification of acute pancreatitis in a prospective cohort: Have all loose ends been tied? Pancreatology. 2014;14:257-62.

16. Talukdar R, Clemens M, Vege SS. Moderately severe acute pancreatitis: Prospective validation of this new subgroup of acute pancreatitis. Pancreas. 2012;41:306-9.

17. Vege SS, Gardner TB, Chari ST, et al. Low mortality and high morbidity in severe acute pancreatitis without organ failure: A case for revising the Atlanta classification to include "moderately severe acute pancreatitis". Am J Gastroenterol. 2009; 104:710-5

18. Mounzer R, Langmead CJ, Wu BU, et al. Comparison of existing clinical scoring systems to predict persistent organ failure in patients with acute pancreatitis. Gastroenterology. 2012; 142:1476-82.

19. Forsmark CE, Baillie J, Practice AG, Economics C, Board AG. AGA institute technical review on acute pancreatitis. Gastroenterology 2007;132:2022-44

20. Aundhakar S, Mahajan S, Agarwal A, Mhaskar D. Acute pancreatitis associated with elevated troponin levels: Whether to thrombolyse or not? Ann Med Health Sci Res. 2013. 3 Suppl 1:S50-2.

21. Dettori JR. Loss to follow-up. Evid Based Spine Care J. 2011; 2:7-10.

22. Kristman V, Manno M, Côté P. Loss to follow-up in cohort studies: How much is too much? Eur J Epidemiol. 2004;19:751-60. 